\title{
Zinc finger protein 668 suppresses non-small cell lung cancer invasion and migration by downregulating Snail and upregulating E-cadherin and zonula occludens-1
}

\author{
XIUPENG ZHANG ${ }^{1}$, GUIYANG JIANG ${ }^{1}$, JINGJING WU ${ }^{1}$, HAIJING ZHOU ${ }^{1}$, \\ YONG ZHANG $^{2}$, YUAN MIAO ${ }^{1}$, YANGYANG FENG ${ }^{1}$ and JUANHAN YU ${ }^{1}$ \\ ${ }^{1}$ Department of Pathology, First Affiliated Hospital and College of Basic Medical Sciences, China Medical University; \\ ${ }^{2}$ Department of Pathology, Cancer Hospital of China Medical University, Shenyang, Liaoning 110001, P.R. China
}

Received August 3, 2016; Accepted August 10, 2017

DOI: $10.3892 / \mathrm{ol} .2018 .7802$

\begin{abstract}
Zinc finger protein 668 (ZNF668) is a recently discovered protein and its expression levels, as well as its involvement in the invasion and metastasis of non-small cell lung cancer (NSCLC), are largely unknown. In the present study, immunohistochemical analysis demonstrated that ZNF668 protein expression was decreased in lung tumors $(51 / 167,30.5 \%)$ compared with adjacent normal lung tissues (43/62, 69.4\%; $\mathrm{P}<0.001)$. Subsequent statistical analysis revealed that ZNF668 expression was negatively associated with increased tumor-node-metastasis stage $(\mathrm{P}=0.019)$ and lymph node metastasis $(\mathrm{P}=0.002)$. Following ZNF668 downregulation by transfection of a ZNF668-expressing plasmid or small interfering RNA, it was demonstrated that ZNF668 inhibited the invasion and migration of NSCLC cells. Furthermore, restoration of ZNF668 expression downregulated the expression of Snail and increased the protein levels of epithelial (E-)cadherin and zonula occludens-1 (ZO-1). The results of the present study suggest that ZNF668 is downregulated in human NSCLC. Furthermore, restoration of ZNF668 expression was demonstrated to decrease the expression of Snail and increase the expression of E-cadherin and ZO-1, suppressing the invasion and migration of NSCLC cells.
\end{abstract}

\section{Introduction}

Adherens junctions and tight junctions are major components involved in the maintenance of epithelial cell stability (1-3).

Correspondence to: Dr Juanhan Yu, Department of Pathology, First Affiliated Hospital and College of Basic Medical Sciences, China Medical University, 77 Puhe Road, Shenyang, Liaoning 110001, P.R. China

E-mail: yujuanhan@foxmail.com

Key words: zinc finger protein 668, zonula occludens-1, epithelial-cadherin, Snail, non-small cell lung cancer, invasion, migration
Adherens junctions are composed of epithelial (E-)cadherin and p120-ctn, $\alpha, \beta$ and $\gamma$-catenin, which are its associated proteins (4-6). Tight junctions involve claudin, occludin and additional components, including the peripheral protein zonula occludens-1 (ZO-1) (7,8). Decreased expression of adherens junction and tight junction proteins is one of the leading causes of tumor invasion and metastasis.

Zinc finger protein 668 (ZNF668) belongs to the kruppel $\mathrm{C} 2 \mathrm{H} 2$-type zinc finger protein family and contains 16 C2H2-type zinc fingers. ZNF668 has been characterized as a breast tumor suppressor gene that regulates p53 stability by binding to the mouse double minute 2 homolog (MDM2) (9). ZNF668 has also been demonstrated to mediate Tip60 acetylation and ionizing radiation-induced hyperacetylation of $\mathrm{H} 2 \mathrm{~A}$ histone family member $\mathrm{X}$ following DNA damage (10). Nevertheless, to the best of our knowledge, the effect of ZNF668 on invasion and metastasis of human tumors has not been determined to date.

In the present study, the expression of ZNF668 was investigated in 167 cases of non-small cell lung cancer (NSCLC) and 62 cases of paired normal lung tissues using immunohistochemistry. Associations with clinicopathological features were also examined. Furthermore, the effect of ZNF668 on invasion and migration and the associated downstream effectors were investigated.

\section{Materials and methods}

Patients. The present study was approved by the local institutional review board of China Medical University (Shenyang, China). Tissue samples were obtained from 167 patients (median age, 60 years; age range, 29-79 years; 107 males and 60 females), who underwent complete surgical excision at the First Affiliated Hospital of China Medical University between April 2010 and August 2012 with a diagnosis of lung squamous cell carcinoma or lung adenocarcinoma. No neoadjuvant radiotherapy or chemotherapy was provided prior to surgery and all patients were given standard chemotherapy following surgery. Of the 167 patients, $55(32.9 \%)$ were treated with platinum-based adjuvant chemotherapy, 18 (10.8\%) underwent platinum-based adjuvant chemoradiotherapy and the other 
94 patients were treated outside (no information of treatment). Histological diagnosis and grading were evaluated according to the 2015 World Health Organization classification of tumors of the lung (11). Tumor staging was performed according to the seventh edition of the International Union against Cancer tumor-node-metastasis (TNM) Staging System for Lung Cancer (12). A total of 73 squamous cell lung carcinoma and 94 lung adenocarcinoma cases, were included. The use of specimens and data was approved by the Ethics Committee of China Medical University and informed consent was written and obtained from the patients prior to participation in the present study. A total of 20 freshly isolated specimens, including tumor and paired normal tissues, were stored at $-80^{\circ} \mathrm{C}$ immediately following resection and used for protein extraction.

Western blotting. Total protein was extracted using a lysis buffer at $4^{\circ} \mathrm{C}$ for $20 \mathrm{~min}$ (Pierce; Thermo Fisher Scientific, Inc., Waltham, MA, USA) and quantified using the Bradford protein assay. A total of $50 \mu \mathrm{g}$ protein/lane was separated via SDS-PAGE on a $10 \%$ polyacrylamide gel. The separated proteins were transferred onto a polyvinylidene fluoride membrane (Merck KGaA, Darmstadt, Germany). The membranes were blocked by $5 \%$ bovine serum albumin (Merck $\mathrm{KGaA})$ at $37^{\circ} \mathrm{C}$ for $2 \mathrm{~h}$. The membranes were then incubated overnight at $4^{\circ} \mathrm{C}$ with the following primary antibodies against ZNF668 (1:100; cat. no. HPA043048; Sigma-Aldrich; Merck KGaA), GAPDH (1:5,000; cat. no. G5262; Sigma-Aldrich; Merck KGaA), snail family transcriptional repressor 1 (Snail; 1:1,000; cat. no. 3895; Cell Signaling Technology, Inc., Danvers, MA, USA), snail family transcriptional repressor 2 (Slug; 1:1,000; cat. no. 9585; Cell Signaling Technology, Inc.), Myc-tag (1:1,000; cat. no. 2276; Cell Signaling Technology, Inc.), Vimentin (1:1,000; cat. no. 5741; Cell Signaling Technology, Inc.), E-cadherin (1:1,000; cat. no. 610181; BD Biosciences, Franklin Lakes, NJ, USA), neural (N-)cadherin (1:1,000; cat. no. 610921; BD Biosciences), Fibronectin (1:1,000; cat. no. 610077; BD Biosciences), $\beta$-catenin (1:1,000; cat. no. 610153; BD Biosciences), $\alpha$-catenin (1:1,000; cat. no. 610193; BD Biosciences), ZO-1 (1:500; cat. no. 21773-1-AP; ProteinTech Group, Inc., Chicago, IL, USA) and Occludin (1:500; cat. no. 13409-1-AP; ProteinTech Group, Inc.). Membranes were washed six times with TBS-Tween-20 and subsequently incubated with horseradish peroxidase-conjugated anti-mouse (1:1,000; cat. no. sc-2954; Santa Cruz Biotechnology Inc., Dallas, TX, USA) or anti-rabbit immunoglobulin G (IgG; cat. no. sc-516087, Santa Cruz Biotechnology) at $37^{\circ} \mathrm{C}$ for $2 \mathrm{~h}$. Protein bands were visualized using electrochemiluminescence (Pierce; Thermo Fisher Scientific, Inc.) and quantified using a bio-imaging system (DNR Bio-Imaging Systems, Ltd., Neve Yamin, Israel)

Immunohistochemistry. Samples were fixed using 10\% neutral buffered formalin at room temperature for $30 \mathrm{~min}$, embedded in paraffin and cut into $4-\mu \mathrm{m}$-thick sections. Immunostaining was performed using the streptavidin-peroxidase method. The antigen retrieval was performed by heating to a temperature of $100^{\circ} \mathrm{C}$ with Citrate buffer (Fuzhou Maixin Biotech Co., Ltd., Fuzhou, China). The sections were then blocked with goat serum (Fuzhou Maixin Biotech Co., Ltd.) at $37^{\circ} \mathrm{C}$ for $1 \mathrm{~h}$. The sections were incubated with a polyclonal rabbit anti-ZNF668 antibodies (1:100; cat. no. HPA043048; Sigma-Aldrich; Merck $\mathrm{KGaA}$ ) at $4^{\circ} \mathrm{C}$ overnight, followed by biotinylated goat anti-rabbit $\mathrm{IgG}$ secondary antibodies at $37^{\circ} \mathrm{C}$ for $30 \mathrm{~min}$ (1:100; cat. no. KIT-9710; Fuzhou Maixin Biotech Co., Ltd., Fuzhou, China). Subsequent to washing three times with PBS, the sections were incubated with horseradish peroxidase-conjugated streptavidin-biotin at $37^{\circ} \mathrm{C}$ for $30 \mathrm{~min}$ (cat. no. KIT-9710; Fuzhou Maixin Biotech Co., Ltd.) and subsequently stained with 3,3-diaminobenzidine tetra-hydrochloride for $1 \mathrm{~min}$ at $25^{\circ} \mathrm{C}$ (cat. no. KIT-0014; Fuzhou Maixin Biotech Co., Ltd.). Finally, samples were counterstained with hematoxylin at room temperature for $5 \mathrm{~min}$, dehydrated in alcohol (the concentration of alcohol ranged between 100 and 70\%), and mounted. Two investigators, blinded to the clinical data, scored the slides semi-quantitatively by light microscopy at x200magnification by evaluating the staining intensity and the percentage of stained cells in representative areas. The staining intensity was scored as 0 , no signal; 1 , weak; 2 , moderate or 3 , high. The percentage of cells stained was scored as 1, 1-25\%;2, 26-50\%; $3,51-75 \%$; or $4,76-100 \%$. A final score of $0-12$ was obtained by multiplying the intensity and percentage scores. Tumors with a score $\geq 4$ were considered ZNF668 positive, whereas a score $<4$ was considered to indicate negative or low ZNF668 expression.

Cell culture. The HBE cell line was obtained from the American Type Culture Collection (Manassas, VA, USA). The A549, H460, H292 and SPC-A-1 (SPC) cell lines were obtained from the Shanghai Cell Bank of the Chinese Academy of Sciences (Shanghai, China). Cells were maintained in RPMI 1640 medium (Invitrogen; Thermo Fisher Scientific, Inc.) supplemented with $10 \%$ fetal bovine serum (Invitrogen; Thermo Fisher Scientific, Inc.), 100 IU/ml penicillin (Sigma-Aldrich; Merck KGaA) and $100 \mu \mathrm{g} / \mathrm{ml}$ streptomycin (Sigma-Aldrich; Merck KGaA). Cells were passaged every two days using a $0.25 \%$ trypsin solution (Invitrogen; Thermo Fisher Scientific, Inc.).

Immunofluorescence analysis. Cells were fixed with $4 \%$ paraformaldehyde at $25^{\circ} \mathrm{C}$ for $30 \mathrm{~min}$, blocked with $1 \%$ bovine serum albumin (Sigma-Aldrich; Merck KGaA) at $25^{\circ} \mathrm{C}$ for $30 \mathrm{~min}$ and incubated with a polyclonal anti-ZNF668 antibody (1:50; cat. no. HPA043048; Sigma-Aldrich; Merck KGaA) overnight at $4^{\circ} \mathrm{C}$, followed by a tetramethylrhodamine isothiocyanate-conjugated secondary goat anti-rabbit IgG antibody (1:100; cat. no. ab6718; Abcam, Cambridge, UK) at room temperature for $1 \mathrm{~h}$. Cells were subsequently counterstained with DAPI at $25^{\circ} \mathrm{C}$ for $5 \mathrm{~min}$. Epifluorescence microscopy was performed using an inverted microscope (TE300; Nikon Corporation, Tokyo, Japan) and confocal microscopy was performed using a laser scanning confocal microscope at x600 magnification in three fields of view (Radiance 2000; Carl Zeiss AG, Oberkochen, Germany).

Plasmid transfection and small interfering RNA (siRNA) treatment. The plasmid concentration was $1 \mu \mathrm{g} / \mu \mathrm{l}$ The pCMV6-ddk-myc and pCMV6-ddk-myc-ZNF668 plasmids were purchased from OriGene Technologies, Inc. (Rockville, MD, USA). ZNF668-siRNA (cat. no. sc-63255) and negative control-siRNA (cat. no. sc-37007) were purchased from Santa 
A

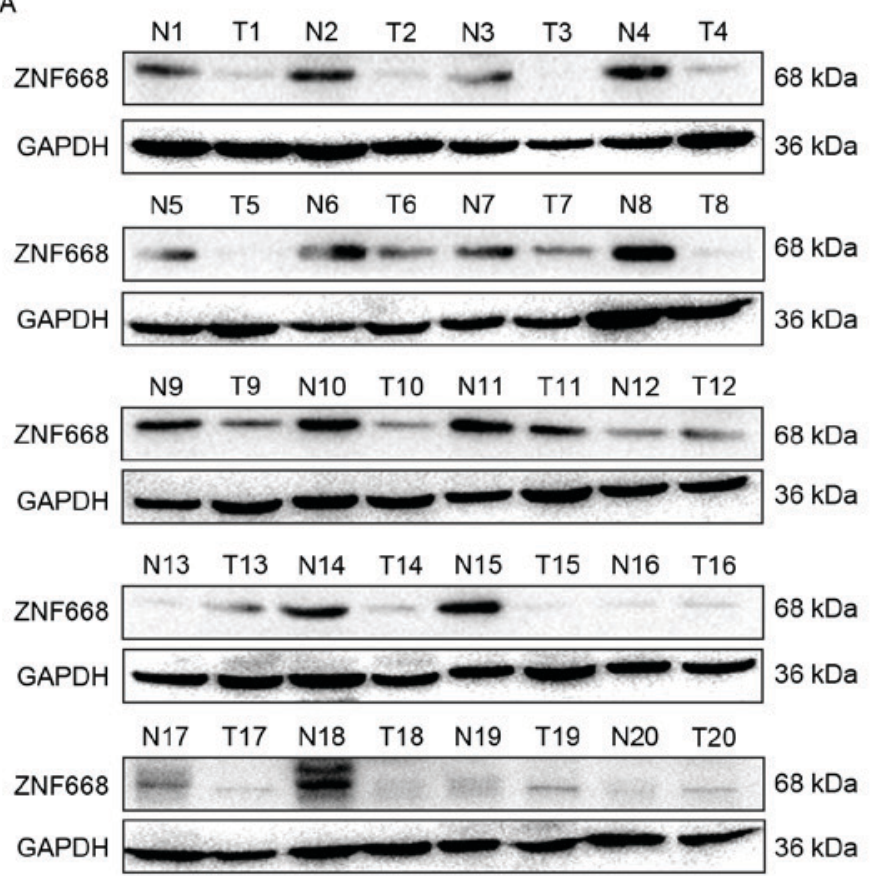

B

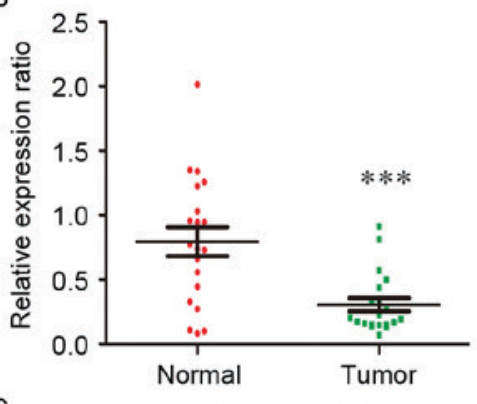

C

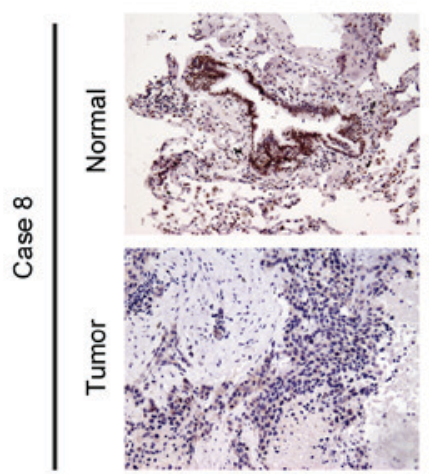

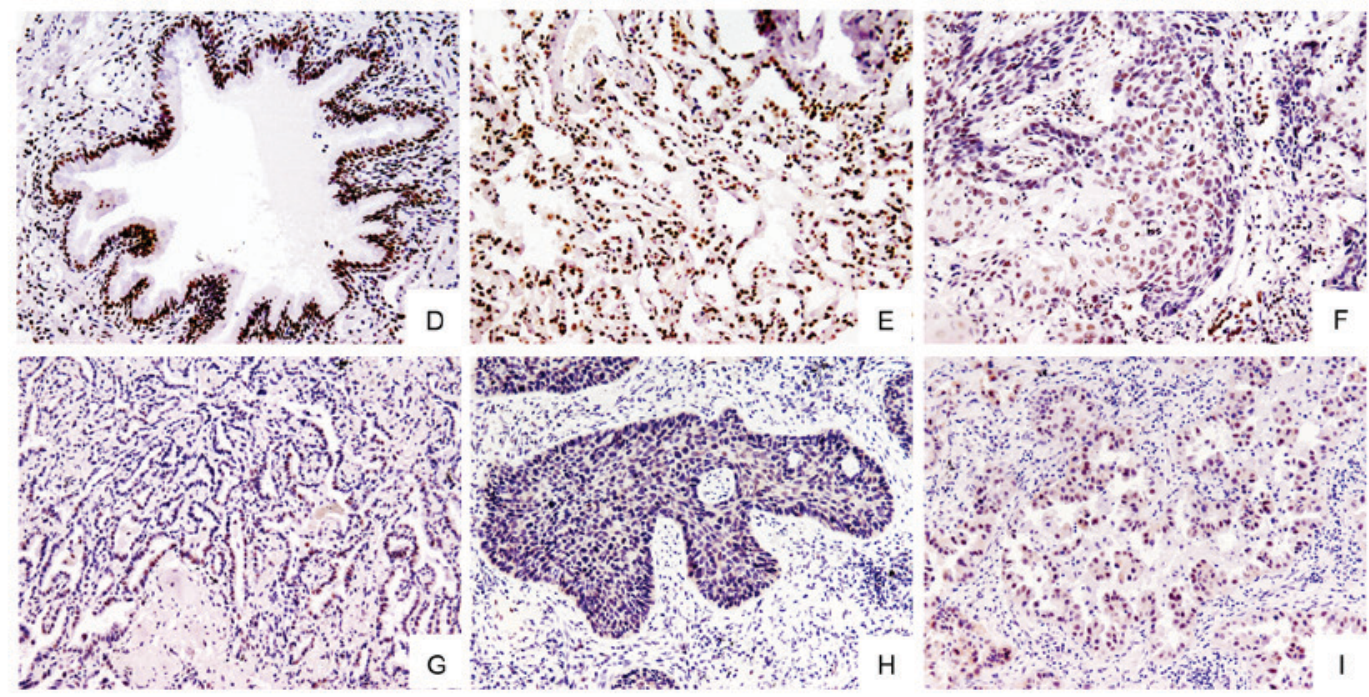

Figure 1. Western blotting and immunohistochemical analysis of ZNF668 protein expression in NSCLC specimens. (A) Among the 20 examined specimens, cases $1,2,3,4,5,6,7,8,9,10,11,14,15,16,17,18$ and 19 demonstrated decreased ZNF668 expression in NSCLC compared with adjacent noncancerous tissues. (B) Quantification of western blotting data revealed significantly decreased ZNF668 expression in NSCLC specimens compared with the corresponding noncancerous tissues. (C) Immunohistochemical analysis of ZNF668 protein expression in paired primary NSCLC and normal lung tissue from a single patient (case 8). ZNF668 immunostaining was positive in the adjacent normal bronchial epithelia, whereas weak ZNF668 expression was observed in the NSCLC sample. Magnification, x200. (D; magnification, x200) Normal bronchial epithelium and (E; magnification, x200) alveoli were positive for ZNF668 Negative or weak nuclear ZNF668 immunoreactivity was observed in (F; magnification, x200) lung squamous cell carcinoma and (G; magnification, x200) adenocarcinoma samples. In certain (H; magnification, x200) squamous cell carcinoma and (I; magnification, x200) adenocarcinoma cases, ZNF668 was localized in the cytoplasm and nuclei. ${ }^{* * * *} \mathrm{P}<0.001$. NSCLC, non-small cell lung carcinoma; ZNF668, zinc finger protein 668; N, normal; T, tumor.

Cruz Biotechnology, Inc. Transfection was performed using the Lipofectamine ${ }^{\circledR} 3000$ reagent (Invitrogen; Thermo Fisher Scientific, Inc.) according to the manufacturer's protocol. The plasmid transfection was harvested at $48 \mathrm{~h}$ after transfection, and the siRNA treatment was harvested at $72 \mathrm{~h}$ after transfection

Matrigel invasion assay. Cell invasion assays were performed using a 24 -well Transwell chamber with $8 \mu \mathrm{m}$ pores (Costar; Sigma-Aldrich; Merck KGaA). The inserts were coated with
$20 \mu 1$ Matrigel (1:3 dilution; BD Biosciences). Following a $48 \mathrm{~h}$ transfection period, cells were trypsinized and a total of $3 \times 10^{5}$ cells in $100 \mu 1$ serum-free medium (RPMI-1640 medium; Invitrogen; Thermo Fisher Scientific, Inc.) were plated in the upper Matrigel chamber for $18 \mathrm{~h}$. Media supplemented with 10\% FBS (Invitrogen; Thermo Fisher Scientific, Inc.) was added in the lower chamber as a chemoattractant. Following incubation at $37^{\circ} \mathrm{C}$ for $18 \mathrm{~h}$, cells that passed through the filter were fixed with $4 \%$ paraformaldehyde at $25^{\circ} \mathrm{C}$ for $30 \mathrm{~min}$ and stained with hematoxylin at $25^{\circ} \mathrm{C}$ for $5 \mathrm{~min}$. Invasive cells were 


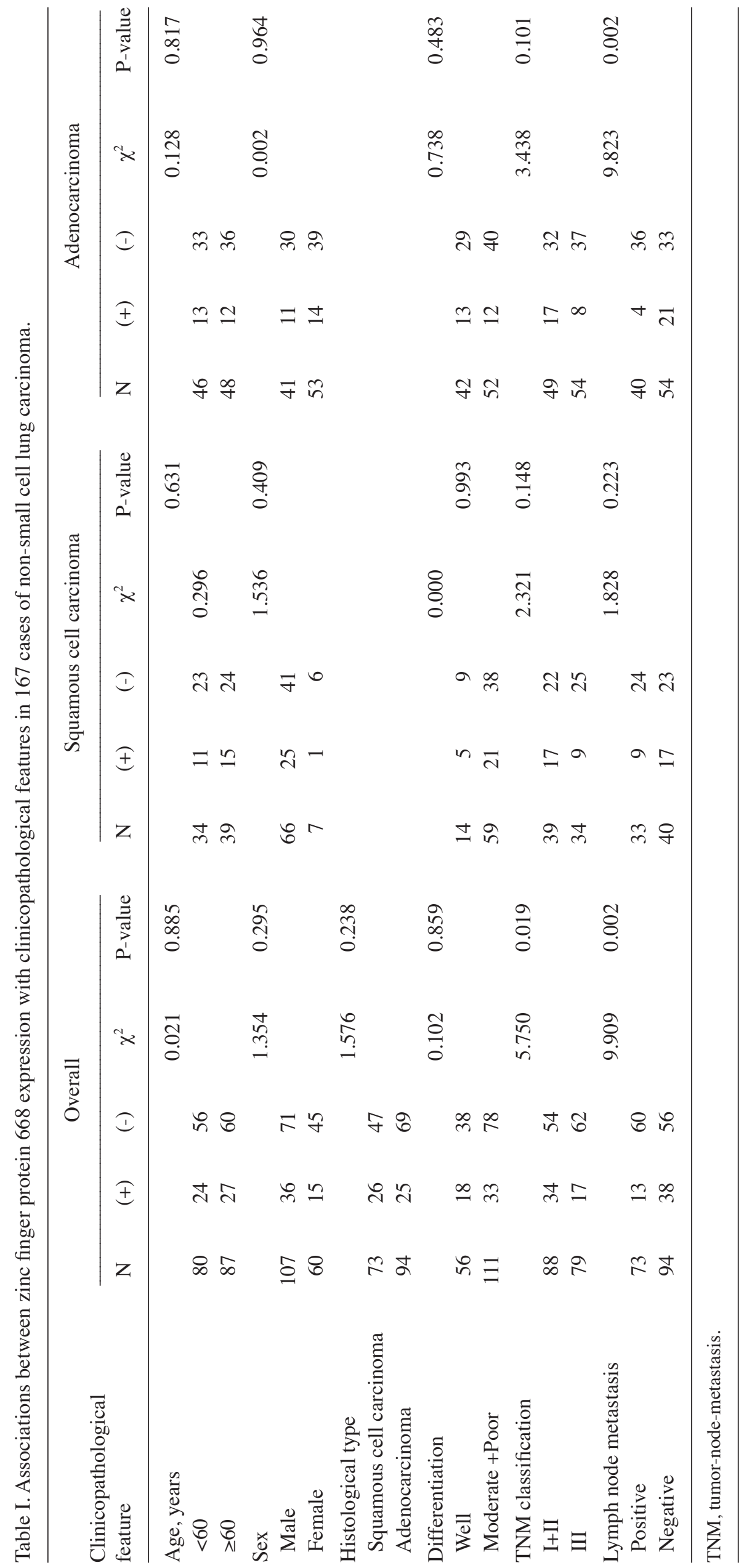




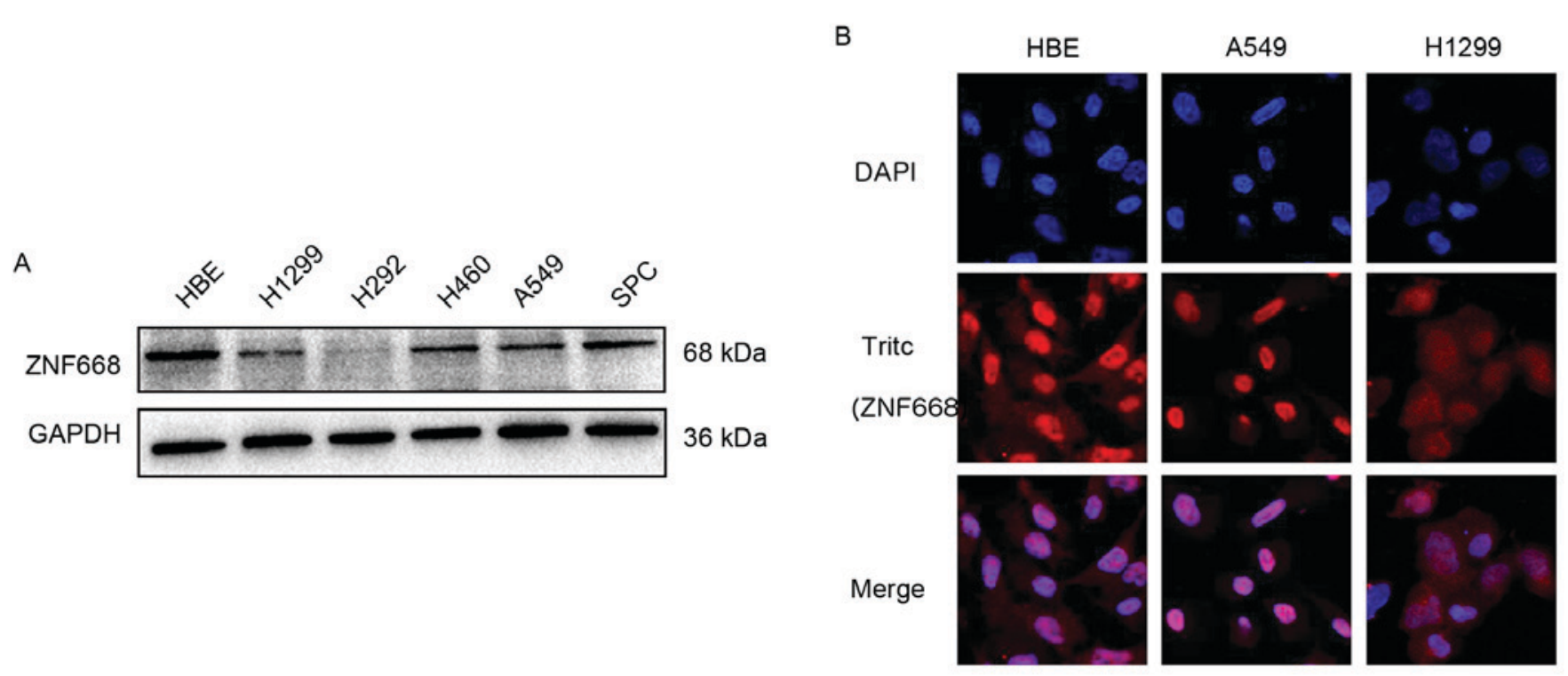

Figure 2.ZNF668 expression in non-small cell lung carcinoma cell lines. (A) Protein expression of ZNF668 in five lung cancer cell lines (A549, H292, H1299, H460 and SPC) and a human bronchial epithelium cell line (HBE). Data are representative of at least three independent experiments generating similar results. (B) Localization of ZNF668 in lung cancer cell lines (HBE, A549, and H1299). ZNF668 was primarily localized in the nuclei of lung cancer cells. Magnification, x600. ZNF668, zinc finger protein 668.

A
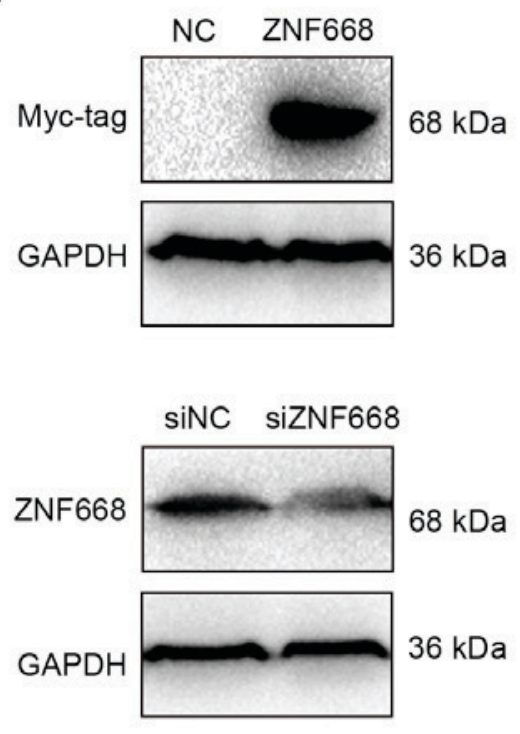

C
B
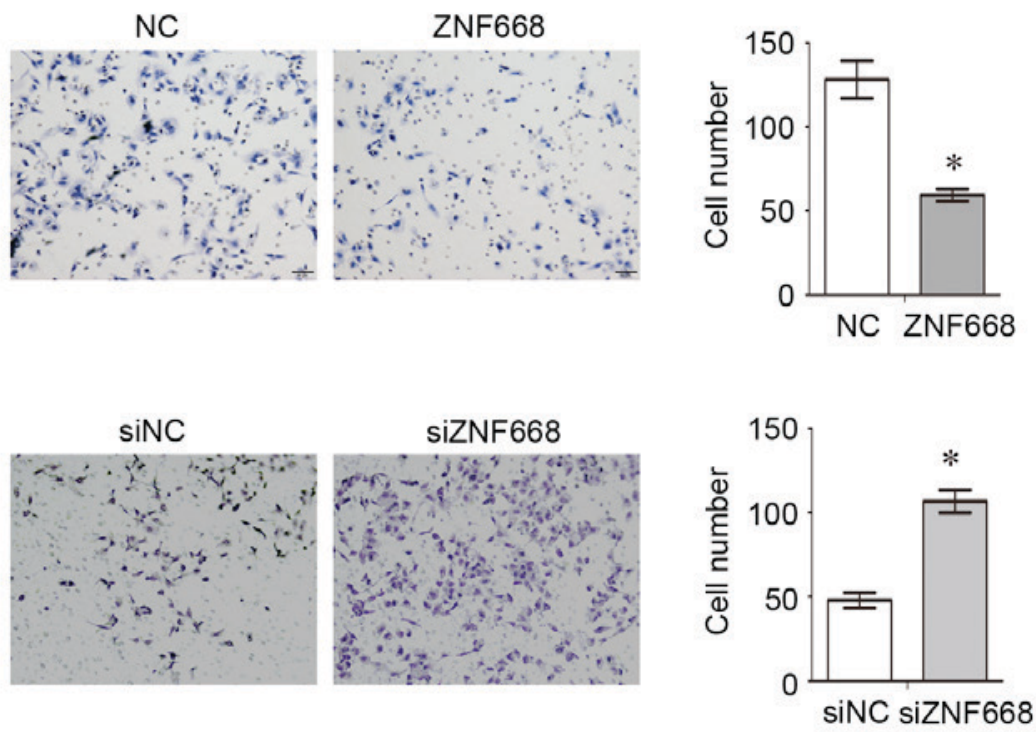

\section{NC (0\&24 h) ZNF668 (0\&24 h)}

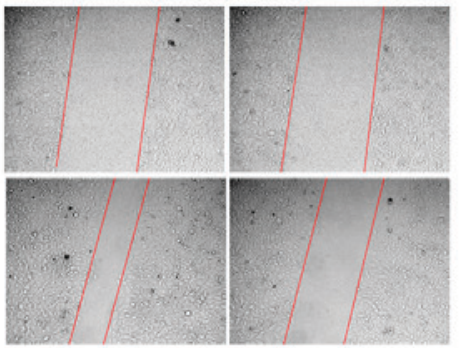

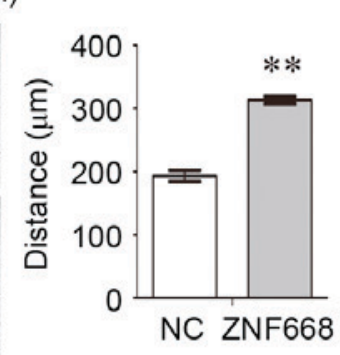

siNC (0\&24 h) siZNF668 (0\&24 h)
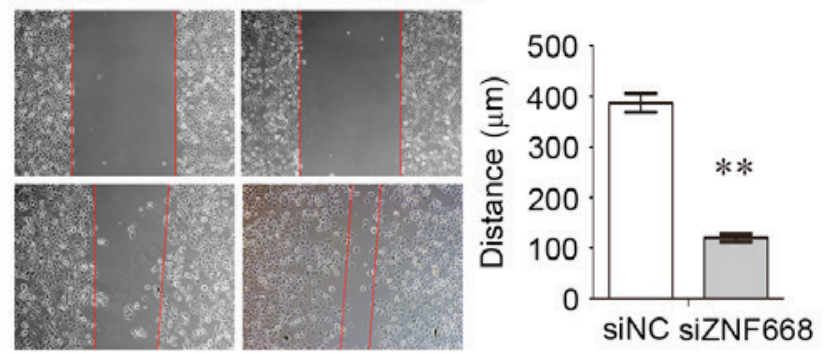

Figure 3. Involvement of ZNF668 in the regulation of lung cancer cell invasion. (A) Western blot analysis demonstrating ZNF668 overexpression or depletion in A549 and HBE cells, respectively (magnification, x600). (B) The average number of migratory cells was counted after $18 \mathrm{~h}$. ZNF668 overexpression resulted in a marked decrease of A549 cell invasion, whereas ZNF668-siRNA treatment significantly promoted cell invasion in HBE cells. (C) Wound healing assay determined at $24 \mathrm{~h}$. ZNF668 overexpression impaired the migration of A549 cells, whereas reduced ZNF668 expression enhanced the migration of HBE cells. Values are presented as the mean \pm standard error of the mean of three independent experiments. ${ }^{* *} \mathrm{P}<0.01$ and ${ }^{*} \mathrm{P}<0.05$ vs. siNC, as assessed with Student's t-test. ZNF668, zinc finger protein 668; NC, negative control; si, small interfering. 
observed by light microscopy at x200 magnification, counted in ten randomly-selected fields.

Wound healing assay. In cultures with cell density $<90 \%$, following a $48 \mathrm{~h}$ transfection period, wounds were created in confluent areas using a $200-\mu 1$ pipette tip. Wound healing within the scrape line was observed at different time points ( 0 and $24 \mathrm{~h}$ ) at $37^{\circ} \mathrm{C}$ and representative pictures for each cell line were captured. Duplicate wells were examined for each condition, and each experiment was performed 3 times. Data were analyzed using Image J $1.48 \mathrm{u}$ Software (National Institutes of Health, Bethesda, MD, USA).

Statistical analysis. SPSS version 17.0 for windows (SPSS, Inc., Chicago, IL, USA) was used for statistical analyses. Pearson's $\chi^{2}$ test was used to analyze the associations between ZNF668 expression and several clinicopathological features. Student's t tests were used for the analysis of western blotting data. $\mathrm{P}<0.05$ was considered to indicate a statistically significant difference.

\section{Results}

ZNF668 protein expression in NSCLC. The expression of ZNF668 in clinical NSCLC samples was initially investigated using western blotting. In lung cancer tissues ZNF668 protein expression was lower compared with the corresponding adjacent noncancerous tissues (17/20; 85\%; Fig. 1A). Analysis of western blotting data indicated a normalized value of $0.7935 \pm 0.1120$ for ZNF668 protein expression in NSCLC specimens, which was significantly lower compared with that of corresponding noncancerous tissues $(0.3057 \pm 0.05185$; $\mathrm{P}<0.001$; Fig. 1B). The subcellular localization of ZNF668 expression in NSCLC and adjacent noncancerous tissues was investigated using immunohistochemistry. A representative paired sample, demonstrating lower ZNF668 expression in the tumor sample, was selected for this analysis. Weak ZNF668 expression was observed in the NSCLC samples (Fig. 1C), in agreement with the western blotting data.

The expression of ZNF668 in NSCLC and adjacent noncancerous tissues was also investigated using immunohistochemistry. ZNF668 was strongly expressed in the nuclei of normal tissues adjacent to carcinomas (Fig. 1D and E); whereas nuclear ZNF668 expression was decreased in lung cancer samples (Fig. 1F and G). Notably, cytoplasmic and nuclear expression was observed in a number of cases (Fig. 1H and I). ZNF668 expression in cancer specimens $(51 / 167,30.5 \%)$ was significantly decreased compared with normal lung tissues (43/62, 69.4\%; P<0.001). As presented in Table I, ZNF668 expression was negatively associated with lymph node metastasis $(\mathrm{P}=0.002)$ and advanced TNM stage $(\mathrm{P}=0.019)$ in NSCLC. No statistically significant associations were identified between ZNF668 expression and sex, age, histological type and differentiation in NSCLC. The association between ZNF668 expression and a number of clinicopathological features in different histological types of NSCLC was also evaluated. As presented in Table I, no statistically significant association between ZNF668 expression and any clinicopathological feature was identified in 73 lung squamous cell carcinoma cases. In 94 lung adenocarcinoma cases, ZNF668

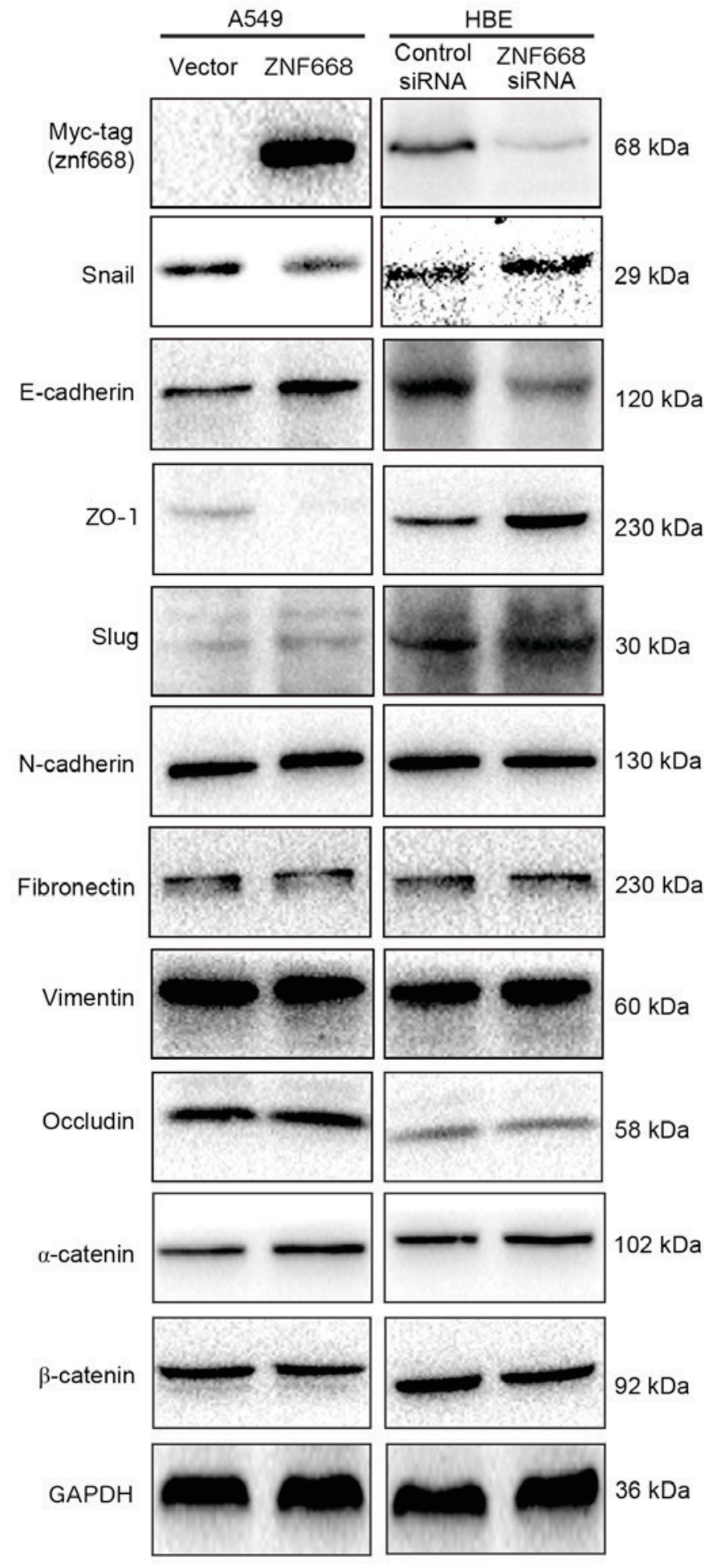

Figure 4. Snail, E-cadherin and ZO-1 expression was dependent on ZNF668 expression in lung cancer cells. Snail expression was decreased, whereas E-cadherin and ZO-1 expression levels increased following ZNF668 overexpression. The opposite effect was observed following ZNF668 silencing. Data represent at least three independent experiments generating similar results. ZNF668, zinc finger protein 668; E-cadherin, epithelial-cadherin; ZO-1, zonula occludens-1; si, small interfering; NC, negative control; N-cadherin, neural cadherin; Slug, snail family transcriptional repressor 2; Snail, snail family transcriptional repressor 1 .

negative expression was significantly associated with lymph node metastasis $(\mathrm{P}=0.001)$. However, no statistically significant association was detected between ZNF668 expression and other factors in NSCLC. 
Expression of ZNF668 in lung cancer cell lines. ZNF668 protein expression was decreased in NSCLC cell lines (5/5) compared with normal HBE cells (Fig. 2A). ZNF668 was primarily localized in the nuclei, whereas weak cytoplasmic expression was observed in A549, H1299 and HBE cells (Fig. 2B).

ZNF668 suppresses NSCLC cell invasion and migration. ZNF668 expression was manipulated by transfecting a ZNF668-expressing plasmid in A549 cells and by siRNA-mediated gene knockdown in HBE cells (Fig. 3A). Transwell assays demonstrated a significant decrease in cell invasion following ZNF668 overexpression, whereas increased invasion was observed following ZNF668 depletion (Fig. 3B). Wound healing assays demonstrated that migration was suppressed in A549 cells overexpressing ZNF668, whereas migration of HBE cells was enhanced when ZNF668 expression was inhibited (Fig. 3C).

ZNF668 downregulates Snail and upregulates E-cadherin and ZO-1 expression. ZNF668 was demonstrated to inhibit the invasion and migration of NSCLC cells. Therefore, the expression of epithelial-mesenchymal transition (EMT)-associated proteins was investigated following ZNF668 overexpression in A549 cells or silencing in HBE cells. ZNF668 overexpression downregulated Snail and increased the expression of E-cadherin and ZO-1. By contrast, ZNF668 depletion resulted in increased Snail expression and decreased E-cadherin and ZO-1 expression levels (Fig. 4). The expression of additional EMT-associated proteins, including occludin, $\alpha$-catenin, $\beta$-catenin, Slug, Vimentin, $\mathrm{N}$-cadherin and Fibronectin, was not altered by ZNF668 manipulation.

\section{Discussion}

The results of the present study demonstrated that ZNF668 is downregulated in human NSCLC, and that ZNF668 overexpression suppresses the migration and invasion of lung cancer cells through the downregulation of Snail and upregulation of E-cadherin and ZO-1.

Due to the fact that ZNF668 is a newly discovered protein, its expression profile and subcellular localization is not well characterized. Using immunohistochemistry, it was demonstrated that ZNF668 is strongly expressed in the nuclei of normal lung tissues, whereas decreased expression was observed in the majority of the tested NSCLC samples. Western blot analysis and immunofluorescent staining in cell lines were consistent with the data obtained from the clinical tissue samples. The results of the present study are in agreement with previously reported data, indicating that ZNF668 primarily has a nuclear localization in breast cancer cells (9). Additional statistical analysis revealed that loss of ZNF668 expression was associated with positive lymph node metastasis and advanced TNM stage in patients with NSCLC; indicating that ZNF668 may serve a critical suppressive function during the development of NSCLC. However, in certain cases, ZNF668 immunoreactivity in the cytoplasm and nuclei was observed. The function of cytosolic ZNF668 expression renders further investigation.
ZNF668 has previously been demonstrated to inhibit p53 degradation by binding MDM2, resulting in suppression of breast cancer cell proliferation (9). However, to the best of our knowledge, its effect on invasion and migration of human tumors has not been thoroughly investigated. The results of the present study demonstrated that overexpression of ZNF668 significantly impaired the invasive and migratory ability of lung cancer cells and resulted in Snail downregulation and E-cadherin and ZO-1 upregulation. Snail directly binds to E-cadherin and suppresses its expression. Previous studies have demonstrated that transforming growth factor- $\beta$, mitogen activated protein kinase, Wnt and phosphoinositide 3-kinase-protein kinase B are the key signaling pathways involved in Snail activation (13-19). Furthermore, Lim et al (20) demonstrated that p53 inhibits the invasion of hepatocellular carcinoma cells by decreasing Snail expression. Further research is required to investigate whether the induced downregulation of Snail following ZNF668 overexpression is associated with the activation of these signaling pathways.

The present study indicated that ZNF668 expression is decreased in NSCLC samples and loss of ZNF668 expression is associated with increased TNM stage and positive lymph node metastasis. Furthermore, ZNF668 overexpression decreases the expression of Snail, while it increases E-cadherin and ZO-1 expression, thus suppressing the invasion and migration of NSCLC cells.

\section{Acknowledgements}

The present study was supported by the National Natural Science Foundation of China (grant nos. 81472805, 81402369 and 81301837).

\section{References}

1. Capaldo CT, Farkas AE and Nusrat A: Epithelial adhesive junctions. F1000Prime Rep 6: 1, 2014.

2. Ivanov AI and Naydenov NG: Dynamics and regulation of epithelial adherens junctions: Recent discoveries and controversies. Int Rev Cell Mol Biol 303: 27-99, 2013.

3. Brooke MA, Nitoiu D and Kelsell DP: Cell-cell connectivity: Desmosomes and disease. J Pathol 226: 158-71, 2012.

4. Liu Y, Xu HT, Dai SD, Wei Q, Yuan XM and Wang EH: Reduction of p120(ctn) isoforms 1 and 3 is significantly associated with metastatic progression of human lung cancer. APMIS 115: 848-56, 2007.

5. Liu Y,Li QC, Miao Y, Xu HT, Dai SD, Wei Q, Dong QZ, Dong XJ, Zhao Y, Zhao C and Wang EH: Ablation of p120-catenin enhances invasion and metastasis of human lung cancer cells. Cancer Sci 100: 441-448, 2009.

6. Liu Y, Wang Y, Zhang Y, Miao Y, Zhao Y, Zhang PX, Jiang GY, Zhang JY, Han Y, Lin XY, et al: Abnormal expression of p120-catenin, E-cadherin, and small GTPases is significantly associated with malignant phenotype of human lung cancer. Lung Cancer 63: 375-382, 2009.

7. Runkle EA and Mu D: Tight junction proteins: From barrier to tumorigenesis. Cancer Lett 337: 41-48, 2013.

8. Balda MS and Matter K: Tight junctions and the regulation of gene expression. Biochim Biophys Acta 1788: 761-767, 2009.

9. Hu R, Peng G, Dai H, Breuer EK, Stemke-Hale K, Li K, Gonzalez-Angulo AM, Mills GB and Lin SY: ZNF668 functions as a tumor suppressor by regulating p53 stability and function in breast cancer. Cancer Res 71: 6524-6534, 2011.

10. Hu R, Wang E, Peng G, Dai H and Lin SY: Zinc finger protein 668 interacts with Tip60 to promote $\mathrm{H} 2 \mathrm{AX}$ acetylation after DNA damage. Cell Cycle 12: 2033-41, 2013.

11. Travis WD, Brambilla E, Burke AP, Marx A and Nicholson AG: WHO Classification of Tumours of the Lung, Pleura, Thymus and Heart. Lyon: International Agency for Research on Cancer, 2015. 
12. Goldstraw P: Updated staging system for lung cancer. Surg Oncol Clin N Am 20: 655-666, 2011.

13. Wu Y and Zhou BP: TNF-alpha/NF-kappaB/Snail pathway in cancer cell migration and invasion. Br J Cancer 102: 639-644, 2010.

14. De Craene B, van Roy F and Berx G: Unraveling signalling cascades for the Snail family of transcription factors. Cell Signal 17: 535-547, 2005 .

15. de Herreros AG, Peiró S, Nassour M and Savagner P: Snail family regulation and epithelial mesenchymal transitions in breast cancer progression. J Mammary Gland Biol Neoplasia 15: $135-147,2010$.

16. Katoh M and Katoh M: Cross-talk of WNT and FGF signaling pathways at GSK3beta to regulate beta-catenin and SNAIL signaling cascades. Cancer Biol Ther 5: 1059-1064, 2006.
17. Julien S, Puig I, Caretti E, Bonaventure J, Nelles L, van Roy F, Dargemont C, de Herreros AG, Bellacosa A and Larue L: Activation of NF-kappaB by Akt upregulates Snail expression and induces epithelium mesenchyme transition. Oncogene 26: 7445-7456, 2007.

18. Kim SO and Kim MR: [6]-Gingerol prevents disassembly of cell junctions and activities of MMPs in invasive human pancreas cancer cells through ERK/NF- $\kappa \mathrm{B} /$ Snail signal transduction pathway. Evid Based Complement Alternat Med 2013: 761852, 2013.

19. Smith BN and Bhowmick NA: Role of EMT in metastasis and therapy resistance. J Clin Med 5 pii: E17, 2016.

20. Lim SO, Kim H and Jung G: p53 inhibits tumor cell invasion via the degradation of snail protein in hepatocellular carcinoma. FEBS Lett 584: 2231-2236, 2010. 